\title{
CICLO EVOLUTIVO DO HEPATOZOON TRIATOMAE (SPOROZOA, HAEMOGREGARINIDAE) PARASITA DE TRIATOMfNEOS
}

\author{
Eduardo Olavo da Rocha e Silva*
}

RSPU-B/277

\begin{abstract}
Rocha E Silva, E. O. da - Ciclo evolutivo do Hepatozoon triatomae (Sporozoa, Haemogregarinidae) parasita de triatomíneos. Rev. Saúde públ., S. Paulo, 9:383-91, 1975.

Resumo: Relata-se o encontro no Estado de São Paulo, Brasil, de triatomineos e lagartos, respectivamente o Triatoma arthurneivai $e$ Tropidurus torquatus, parasitados por uma hemogregarina semelhante a Hepatozoon triatomae (Osimani, 1942) Reichenow, 1953. São apresentados aspectos da sua morfologia $e$ estudadas as diversas fases do seu ciclo evolutivo, este inteiramente reproduzido em laboratório.
\end{abstract}

Unitermos: Sporozoa. Hepatozoon triatomae. Triatoma. Tropidurus.

\section{INTRODUCAO}

Talice $^{10}$ (1929) ao examinar exemplares do Triatoma rubrovaria, foi quem observou pela primeira vez, formas esporozoitas livres, representativas da fase esporogônica de uma hemogregarina, no conteúdo intestinal dos triatomíneos. Foi ainda no Uruguai (Maldonado) que Osimani $^{5}$ (1942) encontrou novos exemplares da mesma espécie, infectados simultaneamente pela hemogregarina e Trypanosoma cruzi, identificando na ocasião o hospedeiro definitivo do esporozoário, um lagarto (Tupinambis teguixin) e classificando o parasita como sendo a Haemogregarina triatomae.

Mais recentemente, no Arizona (USA), Bice $^{1}$ (1965), ao examinar exemplares do Triatoma rubida uhleri, capturados em abrigos de roedores silvestres, os encontrou infectados com hemogragarina, con- siderada pelo autor como a mesma descoberta por Osimani, agora reclassificada como Hepatozoon triatomae (Osimani, 1942) Reichenow, 1953.

Os habitáculos naturais do Triatoma arthurneivai Lent et Martins, 1940, foram descobertos por Corrêa e col. ${ }^{2}$ (1965) ao deparerem com ovos, ninfas e alados da espécie, sob lascas de granito róseo (granito de Itu), nas proximidades da cidade de Sorocaba, Estado de São Paulo. Salientaram no trabalho, a presença nesses ecótopos de grande número de lagartos (Tropidurus torquatus Wied.) e roedores.

A associação alimentar triatomíneo-lacertídeo, especificamente no caso, $T$. arthurneivai $-T$. torquatus, foi sugerida por Forattini e col. ${ }^{3}$ (1968) quando descreveram aspectos do comportamento deste triatomíneo, observado em Votorantim,

* Da superintendencia de Controle de Endemias da Secretaria da Saúde do Estado de São Paulo - Rua Tamandaré, 649 - São Paulo, SP - Brasil. Do Departamento de Epidemiologia da Faculdade de Saúde Pública da USP - Av. Dr. Arnaldo, 715 - São Paulo, SP Brasil. 
ROCHA E SILVA, E. O. da - Ciclo evolutivo do Hepatozoon triatomae (Sporozoa, Haemogregarinidae) parasita de triatomineos. Rev. Saúde públ., S. Paulo, 9:383-91, 1975.

município vizinho a Sorocaba. Ressalte-se que nossas observações identificaram um duplo sentido nesta relação alimentar.

Uma vez caracterizado os ecótopos naturais do $T$. arthurneivai, novos focos da espécie foram paulatinamente detectados nos contrafortes extremos da Serra da Mantiqueira que alcança alguns dos municípios paulistas, situados na região administrativa-5, Campinas: Bragança Paulista, Socorro, Águas de Lindóia, Serra Negra, Pinhal, Santo Antonio do Jardim, Águas da Prata, São João da Boa Vista, Vargem Grande do Sul, São Sebastião da
Grama, São José do Rio Pardo, Divinolândia e Caconde.

A suscetibilidade deste triatomíneo ao $T$. cruzi, embora bem caracterizada em laboratório por Forattini e col. ${ }^{3}$, somente uma vez foi observada na natureza, em que pese as centenas de exemplares examinados (Tabela 1). Foi, aliás, essa procura que possibilitou o encontro da hemogregarina (Rocha e Silva e Pattoli ${ }^{9}$, 1970), objetivando este trabalho, o estabelecimento do seu ciclo evolutivo em laboratório e considerações sobre sua morfologia.

TA BELA 1

Triatoma arthurneivai - exemplares capturados (1969 a 1974)

\begin{tabular}{|c|c|c|c|c|}
\hline \multirow{2}{*}{ Municípios de procedência } & \multicolumn{4}{|c|}{ Número de exemplares } \\
\hline & Capturados & Examinados & Positivos & para T. cruzi \\
\hline Aguas de Lindóia & 10 & 10 & & - \\
\hline Aguas da Prata & 23 & 22 & & - \\
\hline Bragança Paulista & 3 & 2 & & - \\
\hline Caconde & 1 & 1 & & 一 \\
\hline Divinolândia & 280 & 279 & & - \\
\hline Pinhal & 83 & 81 & & - \\
\hline Santo Antonio do Jardim & 46 & 46 & & - \\
\hline São João da Boa Vista & 2 & 1 & & - \\
\hline São José do Rio Pardo & 15 & 15 & & - \\
\hline São Sebastiāo da Grama & 42 & 33 & & $1 *$ \\
\hline Serra Negra & 1 & 1 & & - \\
\hline Socorro & 242 & 220 & & - \\
\hline Vargem Grande do Sul & 2 & 2 & & - \\
\hline Total & 750 & 713 & & $\begin{array}{c}1 \\
(0,14 \%)\end{array}$ \\
\hline
\end{tabular}

MATERIAL E METODOS

Na observação do ciclo evolutivo da hemogregarina na natureza, foram utilizados exemplares do $T$. arthurneivai procedentes do Bairro Lavras de Cima e Fazenda Santo Antonio, Município de Socorro. Sa- liente-se que apenas um exemplar infectado pelo esporozoário foi até agora encontrado em ecótopo artificial; trata-se de um alado macho, capturado numa sala, em uma das casas da fazenda Santo Antonio.

Para o estabelecimento do ciclo experimental em laboratório, foram utilizados 
ROCHA E SILVA, E. O. da - Ciclo evolutivo do Hepatozoon triatomae (Sporozoa, Haemogregarinidae) parasita de triatomineos. Rev. Saúde públ., S. Paulo, 9:383-91, 1975.

exemplares de colônias, alimentadas em aves, mantidas em insetário.

Os T. torquatus encontrados infectados na natureza, procederam unicamente das citadas localidades, capturados entre lascas e vãos de pedra (granito de Itu), juntamente com exemplares de $T$. arthurneivai, a única espécie de triatomíneo observada na área.

Os lacertídeos utilizados nos trabalhos visando completar em laboratório o ciclo evolutivo da $H$. triatomae, procederam de localidades do munióípio de Águas da Prata, onde não foi até então observado o parasitismo em pauta. Em cativeiro, os lagartos foram mantidos vivos durante vários meses, alimentados com larvas de $T e$ nebrio molitor (Coleoptera) e termitas.

O material colhido da luz intestinal dos $T$. arthurneivai, inicialmente era examinado ao microscópio, a fresco em gotas de soro fisiológico, com aumento de 400 vezes. Quando positivo, imediatamente fixado com soro humano inativado e depois de seco, corado pelo May-Grünwald-Giemsa. A hemolinfa era obtida arrancando-se na altura do trocanter uma das patas do par anterior do triatomíneo, gotejada em lâmina bem limpa, para depois de seca ser fixada pelo álcool metílico e corada pelo Giemsa.

O sangue dos lacertídeos, obtido através de punção intracardíaca, era gotejado em lâminas para a preparação de esfregaços finos que depois de secos eram fixados pelo álcool metílico e corados pelo Giemsa (17 minutos). Só então o material era examinado ao microscópio com lente de imersão (1.000X).

Alguns lagartos foram mortos visando a retirada de porções de órgãos internos, especialmente do fígado, pulmões e coração, com os quais por compressão entre lâminas, foram preparadas algumas impressões de órgãos que devidamente coradas pelo Giemsa, se prestaram aos nossos estudos (Tabela 2).
TABELA 2

Tropidurus torquatus, sacrificados, positivos para Hepatozoon triatomae

\begin{tabular}{l|c|c|c|c}
\hline $\begin{array}{c}\text { Identifi- } \\
\text { cacão do } \\
\text { lacer- } \\
\text { tídeo }\end{array}$ & \multicolumn{4}{|c}{ Resultado dos exames } \\
\cline { 2 - 5 } & Sangue & Figado & PuImão & Coracão \\
\hline & & & & \\
\hline
\end{tabular}

Foi com este material, obtido no decorrer de trabalhos que se prolongaram por quatro anos, que realizamos nossas observações sobre as diversas fases do ciclo evolutivo da hemogregarina, de distribuição restrita, cujo hospedeiro intermediário é um triatomníeo silvestre e seu hospedeiro definitivo um lagarto que frequienta o mesmo ecótopo representado pelo espaço situado entre as fendas ou lascas do granito róseo, na região considerada.

\section{RESULTADOS E COMENTÁRIOS}

\section{Fase esquizogônica (Figura 1)}

Nos tecidos de vários órgãos internos do T. Torquatus foram encontrados cistos esquizogônicos da hemogregarina, porém o órgão mais atingido foi sempre o fígado, vindo a seguir os pulmóes. Observou-se dois tipos de cistos: os macrocistos contendo muitos micromerozoitas, denominados por Mackerras ${ }^{4}$ (1962) de esquizontes $\mathrm{X}$ e os microcistos que albergam no seu interior alguns poucos macromerozoitas ou esquizontes $\mathrm{Y}$. Pessoa ${ }^{6,}{ }^{7}$, que vem realizando amplo estudo das hemogregarinas encontradas em serpentes brasileiras, observa não ter sido esclarecida ainda a origem e destino desses cistos. Em nosso material, os cistos esquizogôni- 
ROCHA E SILVA, E. O. da - Ciclo evolutivo do Hepatozoon triatomae (Sporozoa, Haemogregarinidae) parasita de triatomíneos. Rev. Saúde pübl., S. Paulo. 9:383-91, 1975.

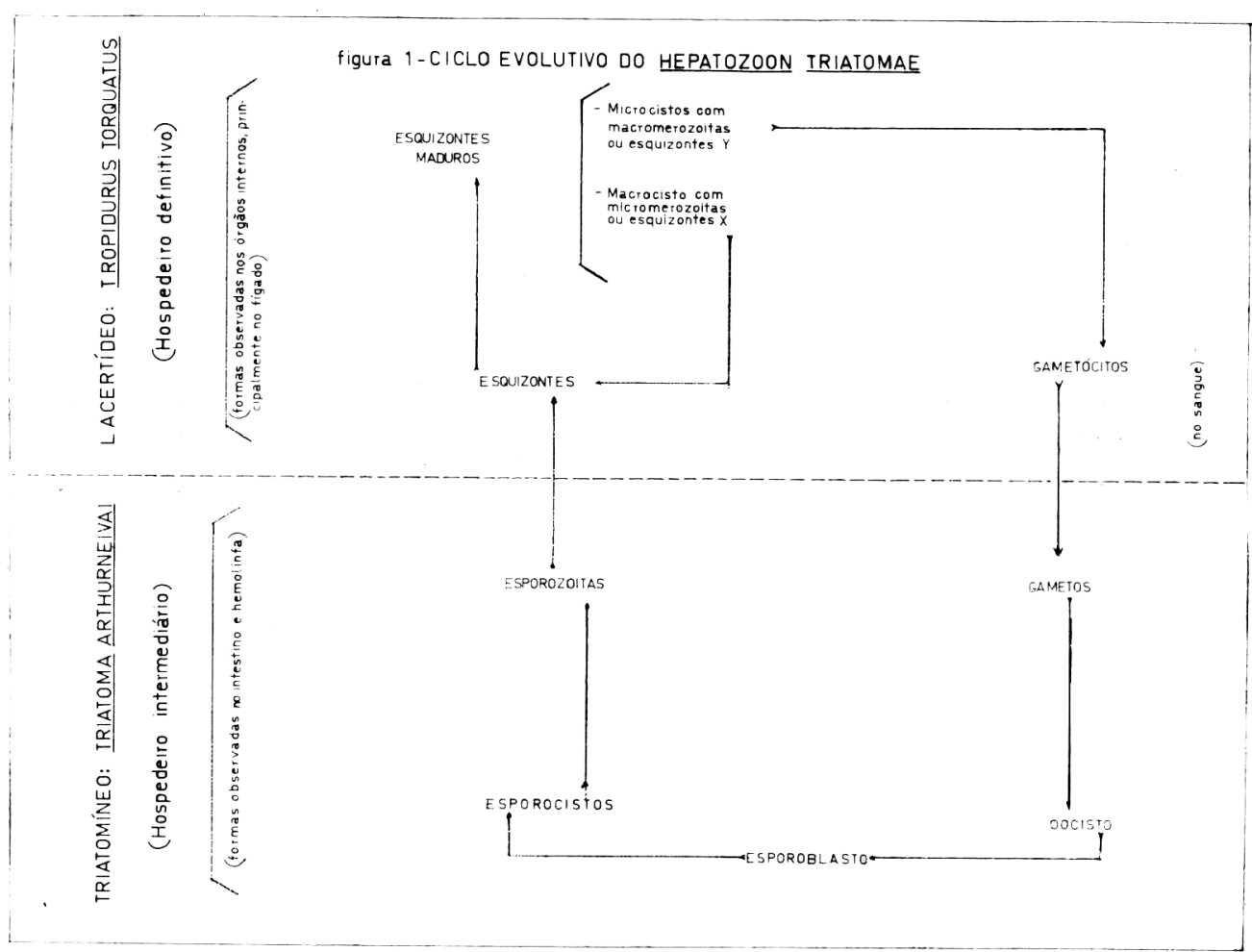

cos foram encontrados nas diferentes fases do seu processo evolutivo: na fase inicial do processo (Fotos 1, 2 e 3), com macroesquizontes bem formados (Foto 4), com microesquizontes (Foto 5) e cistos envolvidos numa cápsula protetora (Foto 6). Observamos também com algumas lâminas, formas livres no fígado, algumas arredondadas ou ligeiramente alongadas (esquizontes tissulares - Foto 7) e outras nitidamente alongadas (gametócitos jovens - Foto 8 ).

No sangue, as formas intraglobulares se apresentam encapsuladas (Foto 9), o que muitas vezes impede a visualização nítida do gametócito no interior. Outras vezes. no entanto, observa-se o gametócito e seu núcleo; este situado no terço médio, apresentando-se ora concentrado com uma coloração riva (Fotos 10 e 11 ), outras rezes menos nítido de tonalidade pálida
(Foto 12). Se isso representa diferenciação de sexo do gametócito, não nos foi possivel apurar. $O$ número de gametócitos presentes no sangue circulante, nas infecções naturais, se situou em torno de um gametócito por 100 a 200 campos microscópicos observados. No início da invasão do sangue pelo parasito e especialmente nas poucas infecções experimentais graves por nós observadas, encontramos formas intraglobulares com morfologia diferente dos gametócitos, fato também observado por Mackerars * (1962) nas hemogregarinas de lagartos australianos e consideradas por ele como sendo esquizontes tissulares (Fotos 13 e 14). Em raríssimas ocasiões foram observadas, também, finas formas extraglobulares, livres no sangue. Quanto às modificações sofridas pelas hemácias, devido à presença da hemogregarina, destaca-se o deslocamento do núcleo 
ROCHA E SILVA, E. O. da - Ciclo evolutivo do Hepatozoon triatomae (Sporozoa, Haemogregarinidae) parasita de triatomineos. Rev. Satide públ., S. Paulo, 9:383-91, 1975.

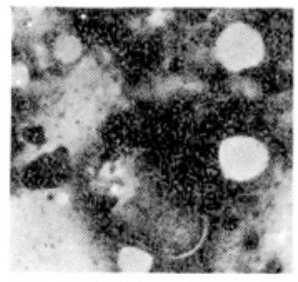

1

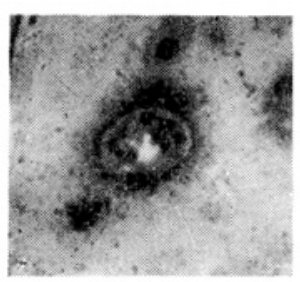

5

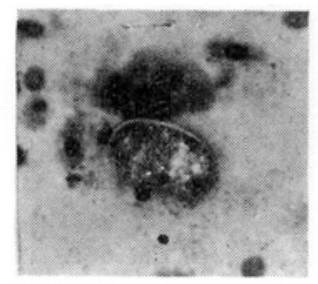

2

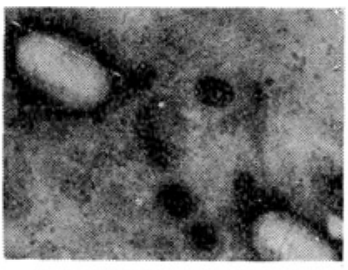

6

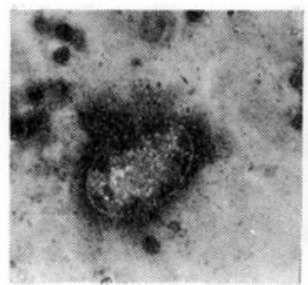

3

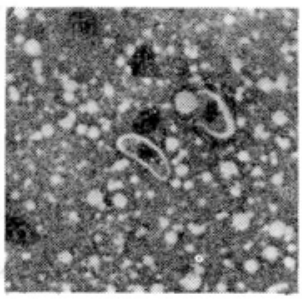

7

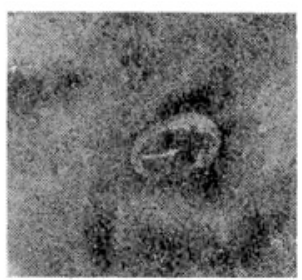

4

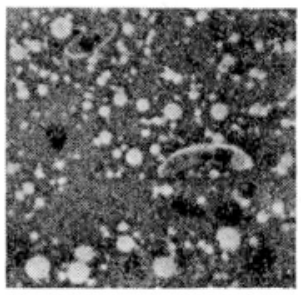

8

Fotos 1. 2 e 3 - Diferentes aspectos do processo de deserivolvimento dos cistos esquizogônicos. Foto 4 - Macroesquizontes visiveis no interior do cisto.

Foto 5 - Microesquizontes visive:s no interior do cisto.

Foto 6 - Cápsula protetora dos cistos.

Foto 7 - Esquizontes tissulares livres.

Foto 8 - Gametócito livre.

para um dos lados ou extremidade (Fotos 9 a 12). A prevalência da infecção no sangue, entre os 112 exemplares do $T$. tarquatus examinados, procedentes das duas localidades citadas, situou-se em torno dos $12 \%$ (Tabela 3 ), variando porém dos vinte e dois ao zero por cento, no decorrer das diversas capturas realizadas entre 1971 e 1974.

\section{Fase esporogômica (Figura 1)}

Vinte e quatro a quarenta e oito horas após a ingestão pelo $T$. arthurneivai, do sangue de lacertídeos apresentando gametócitos intraglobulares, estes já podem ser vistos livres na luz do intestino médio do triatomíneo. Posteriormente, são observados gametócitos em divisão. originando gametas menores (Foto 15), alguns ligeiramente recurvados e outros não. Os ga-
TA B E L A 3

Percentual de infecsão pelo Hepatozoon triatomae, entre os Tropidurus torquatus capturados

\begin{tabular}{l|r|c|c}
\hline \multirow{2}{*}{$\begin{array}{l}\text { Data de } \\
\text { capturas }\end{array}$} & Examinados & \multicolumn{2}{|c}{ Positivos } \\
\cline { 3 - 4 } & & N.o & $\%$ \\
\hline $27-07-71$ & 20 & 2 & 10,0 \\
$18-10-71$ & 18 & 4 & 22,2 \\
$26-10-71$ & 20 & - & - \\
$04-05-73$ & 10 & 2 & 20,0 \\
$09-06-73$ & 22 & 4 & 18,2 \\
$21-11-73$ & 4 & - & - \\
$10-05-74$ & 6 & - & - \\
$11-09-74$ & 12 & 2 & 16,7 \\
\hline Total & 112 & 14 & 12,5 \\
\hline
\end{tabular}

metos tendem a se aproximar como vemos nas Fotos 16 e 17, podendo representar a fase inicial da reprodução pelo processo 
ROCHA E SILVA, E. O. da - Ciclo evolutivo do Hepatozoon triatomae (Sporozoa, Haemogregarinidae) parasita de triatomíneos. Rev. Saúde públ., S. Paulo, 9:383-91, 1975.
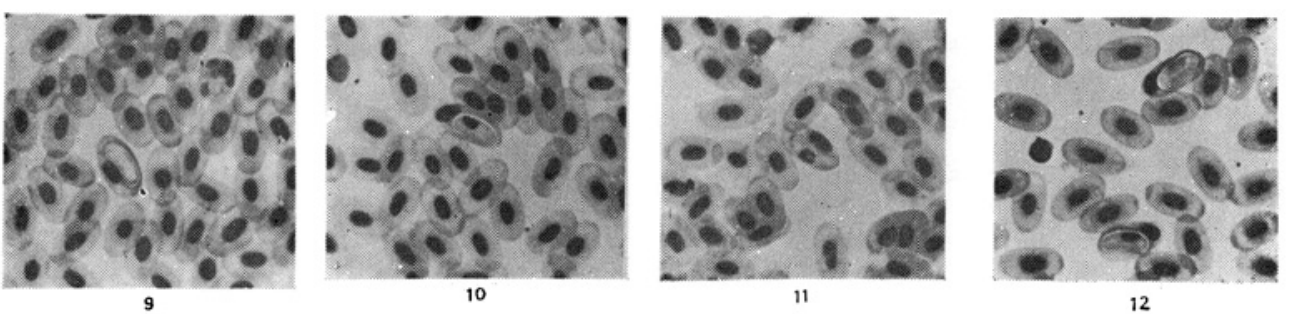

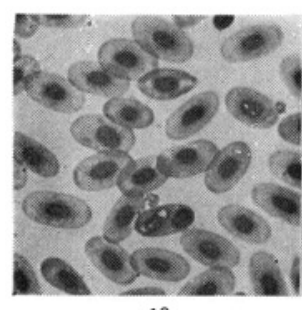

13

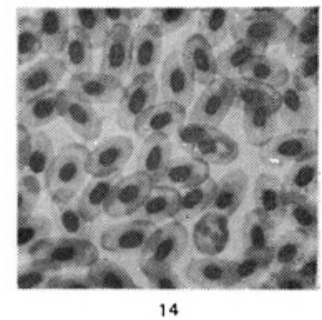

Formas encontradas no sangue do Tropidurus torquatus (aumento de $1.000 \mathrm{X})$ :

Fotos 9, 10, 11 e 12 - Diferentes aspectos dos gametócitos (formas intraglobulares).

Fotos 13 e 14 - Esquizontes tissulares, anormalmente encontrados, no interior dos glóbulos vermelhos (infeccão experimental).

de sisígia, segundo Pessoa ${ }^{s}$. Raramente são observadas formas alongadas, maiores como mostramos na Foto 18. O ovo, quando corado (?) apresenta núcleo bem nítido e vacúolos contendo massas esbranquiçadas separadas entre si por nítidos septos. As membranas externas são pouco visíveis (Fotos 19 e 20). Osimani ${ }^{5}$ também não observou a presença de formas arredondadas com nítida dupla membrana, como as relatadas por Talice ${ }^{10} \mathrm{e}$ fotografadas por Bice ${ }^{1}$.

$O$ s esporoblastos na fase inicial, dificilmente são observados, quer na luz intestinal, quer na hemocele dos triatomíneos. $\mathrm{Na}$ Foto 21, apresentamos como ele é visto a fresco, no intestino, em pequeno aumento.

Os esporocistos são as formas do parasito mais observadas nos triatomíneos, podendo ser encontradas tanto na luz intestinal (maior número) como na cavidade geral, em diferentes fases de desenvolvi- mento e mostrando a fresco a presença de dupla membrana externa (Foto 22). $\mathrm{O}$ número e aspecto dos esporozoitas contidos no seu interior é variável, oscilando em torno dos vinte. 0 mesmo podendo ser dito do número de esporocistos presentes em cada triatomíneo infectado, notando-se, no entretanto, maior número deles, nos exemplares do $T$. arthurneivai menos ingorgitados (Foto 23).

Os esporocistos quando maduros se rompem com facilidade, libertando os esporozoitas (Foto 24). Estes por contrações partidas da região central e dirigidas para as extremidades, movimentam-se lenta porém livremente no meio líquido, sendo vistos em conseqüência, em tamanhos diversos e quase sempre em crescente.

A prevalência da infecção entre os exemplares do $T$. arthurneivai, capturados no bairro Lavras de Cima e fazenda Santo Antonio, foi de aproximadamente $20 \%$ 
ROCHA E SILVA, E. O. da - Ciclo evolutivo do Hepatozoon triatomae (Sporozoa, Haemogregarinidae) parasita de triatomineos. Rev. Saúde públ., S. Paulo, 9:383-91, 1975.
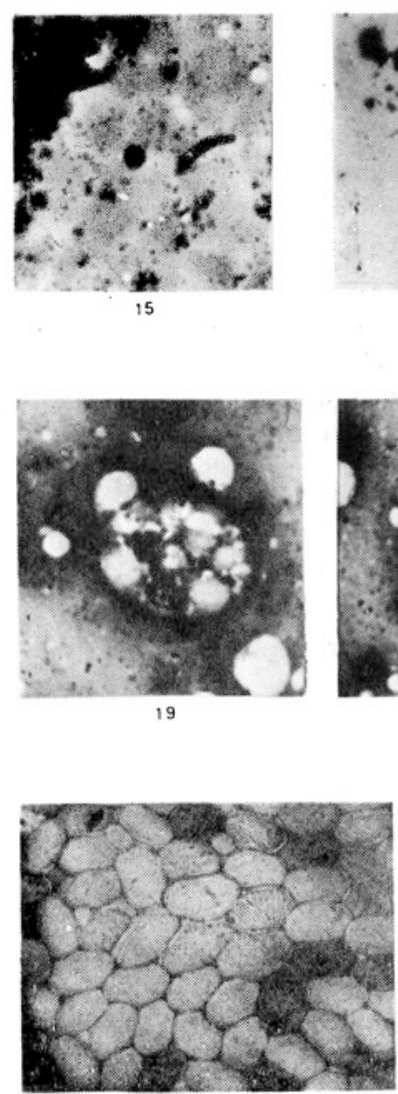

23

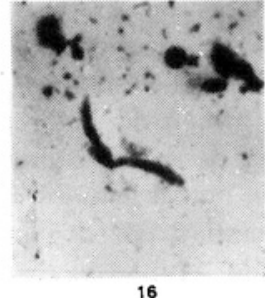

16

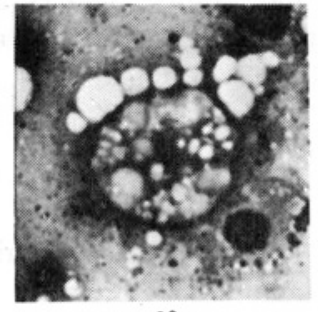

20

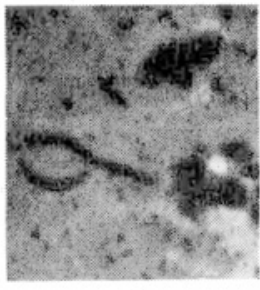

17

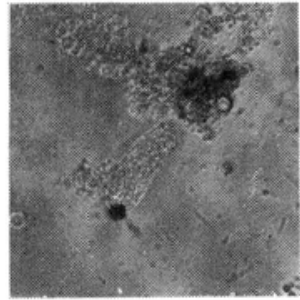

21
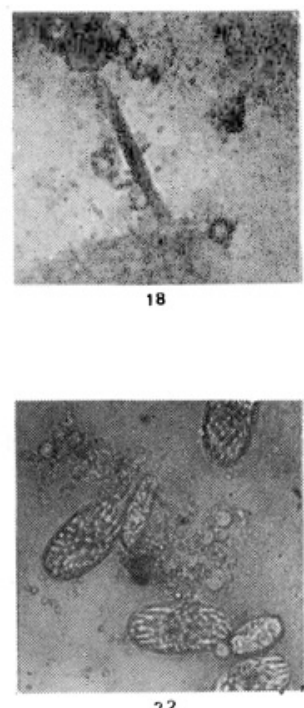

22

Formas no intestino e/ou na hemolinfa do Triatoma arthurneivai (aumento de $1.000 \mathrm{X})$ :

Fotos 15, 16 e 17 - Gametos livres, com tendência a se juntarem.

Foto 18 - Rara forma alongada.

Fotos 19 e 20 - Ovo (?)

Foto 21 - Esporoblasto observado a fresco (aumento de $400 \mathrm{X}$ ).

Foto 22 - Esporocistos a fresco, apresentando dupla membrana externa (aumento de $400 \mathrm{X}$ ).

Foto 23 - Aglomerado de esporocistos corados (aumento de $400 \mathrm{X}$ ). Foto 24 - Esporocisto e esporozoitas livres.

\section{Infecções experimentais}

Em cativeiro, a alimentação de exemplares do $T$. torquatus com triatomíneos, não é fácil de ser obtida. Apesar disso, conseguimos infectar uma dezena de lacertídeos através do repasto em $T$. arthurneivai, infectados com a $H$. triatomae. Saliente-se que esses lagartos foram capturados em localidades limpas e antes do repasto infectante foram examinados mais de uma vez. Decorrido um mês da refeição infectante, já podem ser observados no fígado desses animais os primeiros cistos esquizogônicos. A presença dos primeiros gametócitos intraglobulares no sangue, como já observara Osimani ${ }^{5}$, somente foi constatada ao se completarem 60 dias. De uma maneira geral, a presença dos gametócitos no sangue é precedida pela passagem de esquizontes tissu- 
ROCHA E SILva, E. O. da - Ciclo evolutivo do Hepatozoon triatomae (Sporozoa, Haemogregarinidae) parasita de triatomineos Rev. Salide públ., S. Paulo, 9:383-91, 1975.

lares, extra ou intraglobulares, como se tenta mostrar na Tabela 4.

TABELA 4

Hepatozoon triatomae, periodo prepatente observado nas infeccóes experimentais

\begin{tabular}{|c|c|c|c|}
\hline \multirow{2}{*}{$\begin{array}{l}\text { Identi- } \\
\text { ficação } \\
\text { do lacer- } \\
\text { tideo }\end{array}$} & \multirow{2}{*}{$\begin{array}{l}\text { Processo de } \\
\text { infecção }\end{array}$} & \multicolumn{2}{|c|}{$\begin{array}{c}\text { Periodo decorrido } \\
\text { em dias, para o } \\
\text { encontro de formas } \\
\text { no sangue }\end{array}$} \\
\hline & & $\begin{array}{l}\text { Esquizon- } \\
\text { tes tissu- } \\
\text { lares }\end{array}$ & $\begin{array}{l}\text { Game- } \\
\text { tócitos }\end{array}$ \\
\hline L 15 & via digestiva & 22 & 60 \\
\hline L 18 & via digestiva & 20 & 63 \\
\hline I 19 & via digestiva & 21 & 63 \\
\hline L $14 a$ & via digestiva & $?$ & 60 \\
\hline L 55 & $\begin{array}{c}\text { inoculação } \\
\text { intra-cardíaca }\end{array}$ & $?$ & 30 \\
\hline
\end{tabular}

Aparentemente, a $H$. triatomae causa danos ao hospedeiro vertebrado. Lma possível queda nos níveis imunitários deve ter ocorrido, pelo desencadeamento simultâneo em alguns exemplares, de parasitemias maláricas (Plasmodium tropiduri) anteriormente não observadas, levando um de nossos lagartos à morte, possivelmente em conseqüência da maciça infecção mista constatada.

A infecção dos triatomíneos em laboratório não chegou a ser realizada por Osimani ${ }^{5}$, pelas inúmeras dificuldades que apresentam. Depois de dezenas de tentativas, conseguimos completar o ciclo de desenvolvimento da hemogregarina em duas ninfas do $5 .^{\circ}$ estádio, do T. arthurneivai, alimentadas até então exclusivamente em aves. Elas se infectaram através de repasto realizado em um lacertídeo, procedente de área limpa (município de Divinolândia) e infectado experimentalmente pela ingestão de triatomíneos naturalmente infectados, capturados na fa- zenda Santo Antonio, município de Socorro. No exame realizado 60 dias após a ingestão do sangue infectante, revelaram alto índice de esporocistos maduros e presença de esporozoitas livres. Além dessas duas, outras poucas ninfas se apresentaram infectadas, porém não completaram o ciclo, devido entre outros fatores aos exames realizados. Além do $T$. arthurneivai foram testados exemplares de outras espécies, destas, apenas alguns Rhodnius neglectus chegaram a alimentar nos lagartos.

\section{CONCLUSÖES}

As observações aqui relatadas, fruto não apenas de investigações realizadas com material procedente do campo, mas também de estudos experimentais em laboratório, permite-nos concluir que em duas localidades do município de Socorro Estado de São Paulo, foi constatada a presença de um esporozoário (Adeleida-Haemogregarinidae) parasito cujo ciclo de vida tem como hospedeiro definitivo um lacertídeo, o Tropidurus torquatus Wiedman e como hospedeiro intermediário um triatomíneo, o Triatoma arthurneivai Lent et Martins, 1940.

As características do ciclo evolutivo e os aspectos gerais da morfologia do parasita, nas várias fases, assemelham-se ao da hemogregarina encontrada por Talice $^{10}$ e Osimani ${ }^{5}$, no Uruguai, parasitando - Triatoma rubrotaria e o Tupimambis teguixim. Fotos que nos permitem supor se tratar da hemogregarina reclassificada por Reichenow, como sendo a Hepatozoon triatomae.

\section{A G R A D E C I M EN T OS}

À Diretoria de Combate a Vetores da SLCEN pelas facilidades proporcionadas e aos servidores (auxiliares-técnicos): José de Campos Camargo, Antenor do Nascimento Ferraz Filho e Nuno Lopes Pereira, pela colaboração prestada. 
ROCHA E SILVA, E. O. da - Ciclo evolutivo do Fepatozoon triatomae (Sporozoa, Haemogregarinidae) parasita de triatomíneos. Rev. Saude públ., S. Paulo, 9:383-91, 1975.

RSPU-B/277

Rocha E Silva, E. O. da - [Evolution cycle of the Hepatozoon triatomae (Sporozoa, Haemogregarinidae), parasite of triatominea]. Rev. Saúde públ., S. Paulo, 9:383-91, 1975.

SUMMARY: The author relates the discovery of Triatoma arthurneivai and Tropidurus torquatus, in the localities of Lavras de Cima and Santo Antonio, State of São Paulo, Brazil, infected by a hemogregarine similar to Hepatozoon triatomae (Osimani, 1942) Reichenow 1953. He presents certain aspects of its morphology and also studies the several phases of its evolution cycle, which were completed in the laboratory.

UnITERms: Sporozoa. Hepatozoon triatomae. Triatominae. Tropidurus.

\section{REFERENCIAS BIBLIOGRAFICAS}

1. BICE, D. E. - A sporozoan parasite of Triatoma rubiala uhleri. Rev. Biol. trop., 13:293-6, 1965.

2. CORREA, R. R. et al. - Nota sobre - Triatoma arthurneivai. Seu criadouro extradomicillar. Rev. bras. Malar., 17:217-32, 1965.

3. FORATTINI, O. P. et al. - Dados sobre a biologia do Triatoma arthurneivai no sudeste do Estado de São Paulo, Brasil. Rev. Saude puibl., $\mathbf{S}$. Paulo, 2:186-93, 1968.

4. MACKERRAS, M. J. - The life history of a Hepatozoon (Sporozoa: Adeleidea) of Varanid llzards in Australla. Austr. J. Zool., 10:35-44, 1962.

5. OSIMANI, J. J. - Haemogregarina triatomae n. sp. from a south american lizard. Tupimambis teguixin, transmited by the reduvild Triatoma rubrovaria. J. Parasit., 28:147-54, 1942.

6. PESSOA, S. B. \& BIASI, P. de - Consideraçóes taxonômicas sobre certos esquizogónicos e sobre gametócitos de Hepatozoon (Sporozoa, Haemogregarinidae) parasitas de serpentes brasileiras. Mem. Inst. But., 37: $291-8,1973$

7. PESSOA, S. B. \& BIASI, P. de - Nota taxonômica sobre cistos esporogônicos de algumas espécies de Hepatozoon (Sporozoa, Haemogregarinidae) parasitas de serpentes brasilei-

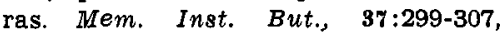
1973.

8. PESSOA, S. B. \& CAVALHEIRO, J. Notas sobre hemogregarinas de serpentes brasileiras. VIII. Sobre a evolução da Haemogregarina miliares na sanguessuga Haementeria lutzi. Rev. bras. Biol., 4:451-8, 1969.

9. ROCHA E SILVA, E. O. da \& PATTOLI, D. B. G. - Considerações sobre a presenca de uma Haemogregarina no tubo digestivo de exemplares de Triatoma arthurneivai. Rev. paul. Med., S. Paulo, 79:91, 1971.

10. TALICE, R. V. - Parasitismo de Triatoma rubrovaria per un sporozoire. Ann. Parasit. hum. comp., ๆ:257-61, 1929.

Recebido para publicação em 06-06-75 Aprovado para publicação em s0-06-75 